\title{
NECESIDADES DE LOS HOMBRES FRENTE A CUIDADOS DE LARGA DURACIÓN DE SUS PAREJAS CON ESCLEROSIS MULTIPLE: LOS CASOS DE FINLANDIA, AUSTRIA Y ESPAÑA
}

\author{
Needs of men in front of the long-term care of their \\ partners with Multiple Sclerosis: the cases of Finland, \\ Austria and Spain
}

M. ${ }^{a}$ CARMEN PÉREZ BELDA*

\section{Resumen}

El papel de los hombres como cuidadores de personas dependientes ha estado omitido o relegado a un segundo plano en el cuerpo de la investigación dedicada al cuidado. La mujer ha sido la gran protagonista dentro de la provisión del cuidado en el ámbito familiar. Aunque durante los últimos años también se han realizado estudios sobre la participación de los hombres en el cuidado y en tareas tradicionalmente realizadas por mujeres.

Este artículo profundiza en las experiencias de los hombres de Laponia, en Finlandia; de Vorarlberg, en Austria; y de Alicante, en España, cuando sus esposas necesitan cuidados de larga duración debido a la enfermedad de Esclerosis Múltiple. Éstas han sido analizadas bajo la perspectiva de género, prestando atención a la influencia de factores contextuales en cada caso. Las relaciones de género, tales como poder, emoción, simbolismo y producción (Connell, 2002), y de las perspectivas interaccional, individual e institucional (Stoller, 2002), han sido la referencia teórica para este análisis. Los resultados de este estudio nos ofrecen la posibilidad de acercarnos

\footnotetext{
* M. ${ }^{a}$ Carmen Pérez Belda Universidad de Alicante Ctra. San Vicente, s/n. 03690 San Vicente del Raspeig (Alicante) Departamento de Trabajo Social y Servicios Sociales Teléfono: +34 965903400 (extensión. 2153) mc.perez@ua.es
} 
a las experiencias que viven los hombres envueltos en el cuidado de larga duración, mostrando la influencia de factores contextuales, tales como los diferentes sistemas de protección social que tienen, así como de factores de género.

Palabras clave: Hombre, cuidados de larga duración, género, Esclerosis Múltiple.

\begin{abstract}
The role of men as carers of dependent people has been omitted or relegated to second place in the body of research devoted to care. Women have been the main protagonist in the provision of care in the family. In the recent years, studies on male involvement in care and tasks traditionally performed by women have also conducted.

This article explores the men's experiences from Lapland in Finland, Vorarlberg in Austria and Alicante in Spain, when their wives need long term care due to Multiple Sclerosis disease. These experiences have been analyzed under the gender perspective, with attention to the influence of contextual factors in each case. Gender relations, such as power, emotion, symbolism and production (Connell, 2002) and interactional individual and institutional perspectives (Stoller, 2002) have been used as reference for the analysis. The results of this study offer the possibility of approaching the experiences that men live with the long-term care situations of their wives, showing the influence of contextual factors, such as the different social protection systems that they since a gender perspective.
\end{abstract}

Keywords: Man, long-term care, gender, Multiple Sclerosis.

\title{
1. Introducción
}

El estudio sobre los hombres como cuidadores ha venido ocupando un segundo plano en el cuerpo de la investigación dedicado al cuidado. Gran parte de estudios previos se ha centrado en la mujer como cuidadora principal en las familias, tales como Durán (2002), La Parra (2002). Sin embargo, en los últimos años se han realizado también diferentes estudios sobre la participación de los hombres en el cuidado desde diversos enfoques, algunos relacionados con el bienestar y la protección social desde una perspectiva de género, feminista o pro-feminista (Pease y Pringle, 2002).

Giddens (2006: 135-163) apunta que Europa vive nuevos retos conectados directamente con la globalización, pero ésta no puede ser vista únicamente como un fenómeno económico. La globalización, de un modo más amplio, debe ser vista más que la integración de actividades económicas, ya que la competitividad en el mercado global es esencial para el futuro de Europa y para la supervivencia del modelo social. Dentro de este modelo social, los cambios y la diversidad de las estructuras familiares traen consigo nuevos retos en el ámbito del cuidado, y con ello una mayor evidencia de la participación de los hombres en este campo tradicionalmente relegado a las mujeres. La 
transformación de la estructura familiar es uno de esos cambios. Las nuevas tendencias son complejas pero tienen una fuerte influencia en el cuidado y en los roles desarrollados por las familias. Kramer (2002: 3) sugiere que se producirá un incremento del número de hombres cuidadores durante o a través del curso de la vida debido al aumento de la longevidad de la población, por lo tanto necesitamos un mejor entendimiento de esas tendencias desde el punto de vista de todos los profesionales y disciplinas para ofrecer respuestas efectivas.

Las necesidades que los hombres experimentan cuando se ven envueltos en situaciones familiares de cuidados de larga duración, especialmente cuando la persona enferma es su pareja, son muy diversas y están influenciadas en gran medida tanto por factores contextuales, culturales, políticos, económicos etc., como por factores personales. Estos factores personales tienen relación con el grado de adaptación que sufren frente a los cambios, dependiendo del contexto en el que viven. Algunos de estos esposos o parejas comienzan a realizar prácticas que hasta ese momento eran desarrolladas únicamente por sus esposas, además, también encuentran dificultades en el desarrollo del cuidado personal (bañar, asear, llevar al baño, etc.). Por lo tanto, las vivencias varían dependiendo del grado de aceptación/resignación o negación de la situación, así como del apoyo recibido.

De esta manera, y basándome en el estudio realizado en mi tesis doctoral sobre las experiencias de los hombres, esposos o parejas, con mujeres en situación de cuidados de larga duración debido a la enfermedad de Esclerosis Múltiple, en Finlandia, Austria y España, pretendo profundizar y explorar en las necesidades experimentadas por estos hombres y sus familias, algunas comunes a todos los casos independientemente de su procedencia según apuntan los resultados. De forma preliminar, los resultados indican que la participación de los hombres en las tareas tradicionalmente realizadas por mujeres viene influenciada por las relaciones de género y el contexto en el que viven. Del mismo modo, las necesidades experimentadas estos hombres pueden diferir dependiendo de factores contextuales, relacionados directamente no sólo con la cultura o con las prácticas, sino también con el apoyo institucional que puedan percibir, y con el objetivo de facilitar o fomentar su rol como cuidador formal o informal. Estas necesidades influirán a su vez en otras necesidades que señalo en este artículo como personales y que van relacionadas con los sentimientos y cambios experimentados por los hombres.

El objetivo de este artículo es alcanzar un mayor entendimiento de las experiencias de estos hombres, prestando atención a qué hacen y a cómo se sienten envueltos en la situación de cuidado cuando sus mujeres necesitan 
cuidados permanentes. Se pretende detectar las necesidades experimentadas por los casos estudiados en estos tres países, cada uno con un sistema de protección social diferente, porque para comprender y alcanzar igualdad en el cuidado a dependientes es necesario abrir un debate sobre las prácticas que los hombres realizan en este campo y el apoyo que reciben ante los nuevos cambios que la sociedad presenta. Para ello, se intenta encontrar respuesta a lo largo de esta investigación a dos cuestiones importantes: ¿cuáles son las necesidades institucionales o de apoyo formal detectadas por los esposos entrevistados en Laponia, Vorarlberg y Alicante durante el proceso de la enfermedad? y ¿cuáles son las necesidades personales que los hombres y esposos o parejas expresan de manera implícita o explícita durante el proceso de la enfermedad?

\section{Conceptualización de cuidado y aspectos generales del hombre como cuidador}

Para comenzar este punto es necesario mencionar la importancia y derecho que tienen las personas y las familias de enfermos con enfermedades crónicas a una calidad de vida, así como a las oportunidades para su desarrollo humano, como bien indica Martínez Román (2002: 145).

Tradicionalmente, la labor de los hombres ha estado relacionada con la esfera pública de la vida, ocupándose del sostenimiento económico del núcleo familiar, mientras que la labor de las mujeres ha estado relacionada con las responsabilidades del cuidado de la familia de manera no remunerada, incluyendo el cuidado a mayores, miembros con discapacidad, hijas/os. Además, cuando de mujeres incluidas en el mercado laboral hablamos, el trabajo a tiempo parcial ha sido la modalidad de empleo en la que las mujeres aparecen con mayor incidencia que los hombres. Esto ha significado que la mayoría de los estudios realizados acerca de personas que se ocupan del cuidado de dependientes se centrará en resaltar la feminización de esta labor, omitiendo el trabajo y las experiencias que algunos hombres realizan, aunque ésta fuera minoritaria. Sin embargo, considero que es importante prestar atención al papel desarrollado por algunos hombres como cuidadores con el fin de mostrar y abrir debate sobre las prácticas que realizan, puesto que para lograr la igualdad en materia de cuidado, que no de conciliación, sino de corresponsabilidad, es necesario observar las necesidades de cambio que la sociedad va experimentando, así como explorando y delimitando el cuidado, su tipología, pero también su calidad, cuestiones que dependerán en gran medida de la propia construcción de género. 
Pero para poder comprender mejor las experiencias de los hombres con mujeres en situación de cuidado definiré en un primer lugar qué entendemos por cuidado. La autora finlandesa Zechner (2008: 33) indica que el cuidado abarca trabajos concretos de cuidados, desde la observación para detectar las necesidades del cuidado, a la responsabilidad de iniciar y mantener actividades de cuidado, para lo que son necesarios los recursos y el tiempo suficiente, sin olvidar tampoco las actividades de cuidado referidas al cuidado mental y emocional de la persona. Pero hablar de cuidado de forma genérica no es suficiente cuando hablamos de la enfermedad de Esclerosis Múltiple. En estos casos el cuidado es requerido durante décadas, por lo tanto, sería más preciso si hablamos de cuidados de larga duración. A este respecto, Stone (2001: 97) lo define como un tipo de cuidado que abarca un amplio rango de asistencia que las personas con algún tipo de discapacidad crónica necesitan durante un periodo de tiempo prolongado, debido a la pérdida de su funcionamiento físico o mental.

La Parra (2002: 34-88) apuntó que la manera en que una sociedad organiza la respuesta informal del cuidado, depende del proceso de construcción de género, de la organización del mercado laboral y del Estado de Bienestar. Por lo tanto, las necesidades que los esposos objeto de este estudio puedan experimentar depende del apoyo que puedan recibir en relación al tipo de Estado de Bienestar que disfruten, perteneciendo cada uno de los países a un modelo de Estado de Bienestar diferente, así como del rol que juegue en ello la organización del mercado laboral. Este contexto influirá a su vez en las prácticas que los hombres realizan influenciadas por las relaciones de género en todos los niveles, porque como bien indica Connell (2002), el concepto de género no es algo estanco, sino que cambia y se va creando día a día con las prácticas que realizamos.

Una vez definido el concepto de género es necesario mencionar brevemente varios aspectos destacables de los estudios de los hombres como cuidadores principales. En primer lugar, Parker y Seymour (1998: 187-191) destacan que las mujeres se involucraban de una manera más intensa en el cuidado que los hombres en la misma situación y que los hombres están menos dispuestos a proporcionar cuidado personal que las mujeres, entendiendo por cuidado personal a la ayuda necesaria para bañarse, vestirse o ir al baño, entre otras tareas de la vida diaria, considerando que un cuidador del mismo sexo sería más apropiado para realizar dichas tareas. Además, en los resultados se destaca también que la edad y el matrimonio como estado civil son las principales variables para que los hombres se conviertan en cuidadores, más concretamente que el estado marital, la combinación de la convivencia y las 
relaciones íntimas previenen a los hombres de escaparse de las responsabilidades de cuidado.

Por otro lado, Detinberg y Clarkberg (2002: 873-874) coinciden en la importancia de la relación de la pareja para la participación de los hombres en el cuidado, es decir, destacan que la naturaleza de la experiencia del cuidado depende en gran medida del grado de la naturaleza de la relación entre el cuidador y la persona cuidada, particularmente en el caso de una pareja o matrimonio. De este modo, mientras unos hombres se involucran más en el cuidado, otros eluden las necesidades de cuidado de las esposas o persona enferma como, por ejemplo, a través del retraso de su jubilación, y se centran en las necesidades económicas de la familia con el fin de huir o escapar de la situación de cuidado, proyectando la lealtad a la vida laboral, lo que paradójicamente les conlleva a una menor satisfacción de su rol como trabajador.

\section{Metodología}

\subsection{Selección de casos con Esclerosis Múltiple}

La Esclerosis Múltiple (EM) es una enfermedad del sistema nervioso central impredecible que se desarrolla durante un largo periodo de la vida. Concretamente, es una condición inflamatoria y de «desmielinización». La mielina es un material que cubre y aísla los nervios, permitiendo la transmisión de los impulsos de forma rápida. La Esclerosis Múltiple afecta a una población comprendida entre los 15-55 años, aunque en la mayoría de los casos aparece entre los 25-30, y afecta en un $60 \%$ a las mujeres. La esperanza de vida es de al menos 25 años desde la aparición de la enfermedad y la mayoría de los pacientes mueren por causas no relacionadas con la misma (Fernández y Fernández, 2004: 13-38).

El motivo de la elección de estudiar los casos en los que la pareja sufre Esclerosis Múltiple ha permitido encontrar más fácilmente casos donde los hombres son cuidadores, ya que según estudios como Parker y Seymour (1998) el hombre participa más en el cuidado cuando la persona enferma es la esposa o pareja. Además, estos hombres se encuentran todavía en edad activa por lo que su participación y experiencias en el cuidado a sus parejas o esposas conllevan muchos cambios, tanto económicos como emocionales, en la vida familiar y de pareja. Otros autores, como Fink (2004: 7), remarcan que no es posible presumir que la participación de los hombres en el cuidado de sus parejas ocurra en todos los casos, y que los esposos o parejas participan en el cuidado del otro de igual manera y con las mismas habilidades, especialmente en lo referente al cuidado personal. Parker y Seymour (1998: 187) apuntaron 
los problemas que los hombres experimentaban en las relaciones con sus parejas a la hora de realizar el cuidado personal, entendiendo este relacional con tocamientos para el aseo, desnudez o contacto con excrementos, entre otros.

El curso de esta enfermedad varía en cada caso. Las personas afectadas pueden sufrir periodos de ataques y otros de remisión. La recuperación de esos ataques también difiere en cada caso pudiendo ser parcial o total. Sin embargo, otros pacientes pueden sufrir un deterioro gradual, o experimentar uno o dos ataques o momentos de crisis en el momento del diagnóstico y no volver a sufrir más síntomas durante años, aunque cuando aparezca después de ese tiempo sea de modo gradual. Por lo tanto, el curso tan diferente de la enfermedad en cada persona ha hecho que el estudio se base, independientemente del tipo de EM que se pueda padecer, en el grado de independencia que tenga la persona afectada, puesto que no todas las personas diagnosticadas pueden tener las mismas necesidades de cuidado. Para ello, se ha realizado una simple división de los casos objeto de estudio, caracterizándolos como independiente, cuando no necesita ayuda para las actividades cotidianas, pero sin embargo no puede trabajar debido a otros síntomas como, por ejemplo, el cansancio; semiindependiente, cuando necesita cierto cuidado o ayuda para las tareas cotidianas, pero no necesita el cuidado de una persona permanentemente; y dependiente, cuando necesita ayuda constantemente.

\subsection{Recogida de información}

La decisión de elegir los países de Finlandia, Austria y España se debe a los diferentes sistemas de protección social y bienestar social que tienen. En este estudio de caso múltiple he entrevistado a tres parejas en Finlandia, Austria y España, concretamente en las regiones de Laponia, Vorarlberg y Alicante. Para estudiar y recabar información sobre las prácticas de los hombres y sus experiencias en las situaciones de cuidado de larga duración de sus esposas, se ha utilizado la entrevista cualitativa semiestructurada. Las entrevistas han sido conducidas y realizadas en la lengua materna de los entrevistados (finés, alemán y español). La colaboración de intérpretes ha sido necesaria para la realización de las entrevistas en Finlandia y Austria. La mayoría de los hombres entrevistados estaban casados, y todos vivían junto a sus parejas en el hogar familiar, requisito imprescindible para poder participar en el estudio. Todos los hombres se encuentran en una edad activa, y la mayoría trabaja fuera del hogar, aunque este hecho cambia de un país a otro, ya que en Finlandia, los hombres pueden recibir una prestación económica por cuidar a la persona enferma y convertirse en cuidadores formales de sus esposas. 
Las diferentes asociaciones o grupos de autoayuda han sido esenciales para encontrar casos dispuestos a participar en esta investigación. Primordialmente, los primeros contactos se hicieron a través de las presidentas de estos grupos de autoayuda, en los casos de Finlandia y Austria, y a través de la trabajadora social de la Asociación de enfermos de Esclerosis Múltiples de Alicante, en España. La ayuda de traductores a la hora de realizar las entrevistas fue totalmente necesaria, con el fin de que las personas entrevistadas pudieran expresarse en su propia lengua materna. Además, también se requirió ayuda para las transcripciones y traducciones de las mismas, ayuda que fue ofrecida por los departamentos de Trabajo Social de la Universidad de Laponia y la Universidad de Ciencias Aplicadas de Vorarlberg. Pero para el estudio de las necesidades y experiencias de los hombres entrevistados, el análisis de contenido ha sido el método utilizado.

\section{Resultados}

\subsection{Las necesidades institucionales detectadas por los hombres en las situaciones de cuidados de larga duración}

Los resultados de este estudio apuntan diferentes necesidades que se han catalogado como institucionales, y son referidas a la responsabilidad institucional que cada uno de los sistemas de protección social tienen en los casos entrevistados. En primer lugar, los resultados apuntan una falta de información en el momento del diagnóstico en los tres países o regiones, tanto de la propia enfermedad como de los servicios accesibles a las mujeres enfermas de Esclerosis Múltiple y a sus familiares. Otro de los aspectos importantes es la falta de coordinación entre los servicios sociales y los sanitarios, concretamente en los casos de Austria y España. Justamente en estos países es donde aparece una menor presencia de la participación de los hombres en el cuidado de sus esposas. En el caso español, los esposos no participan en el cuidado de los casos independiente y dependiente, y en el caso austriaco tampoco lo hace el esposo del caso semiindependiente.

Los tres entrevistados en Vorarlberg (Austria) muestran cómo una vez diagnosticada la enfermedad y percibida la prestación por cuidados de larga duración, no existe un seguimiento ni coordinación entre los servicios sanitarios y sociales, a pesar de las posteriores visitas al hospital debido a las recaídas. La falta de seguimiento y coordinación cuando los pacientes abandonan el hospital entre los servicios de salud y los municipales se hace visible en los casos entrevistados. Además, esto influye en la calidad de los cuidados, la cual no puede asegurarse como tampoco la sobrecarga que los familiares a cargo 
de la persona enferma encuentran. Esto es debido a que, en la mayoría de casos, el dinero obtenido por la prestación de cuidados de larga duración que percibe la persona enferma, cuya cuantía depende del grado de dependencia y que tiene por objeto cubrir los gastos de los cuidados necesarios, es guardado por la familia, y es esta la que se ocupa de la atención de las mujeres enfermas. Por consecuente, la percepción de esta prestación económica no asegura una calidad del cuidado ni tampoco un seguimiento.

Un ejemplo de la falta de calidad en el cuidado se observó en el caso dependiente austriaco en el que la mujer dependiente no recibía ningún tipo de aseo personal, baño o ducha, permaneciendo sola toda la mañana sentada en su silla de ruedas mientras el esposo estaba en el trabajo, es decir, durante un periodo de tiempo aproximado de tres horas en el que ella se intentaba asear. Además, la respuesta de la mujer a la pregunta sobre quién le ayuda a bañarse fue que no sudaba y por lo tanto no necesitaba bañarse, sólo en verano su esposo la metía en la piscina del jardín cuando sudaba y hacía mucho calor. Este claro ejemplo muestra la falta de calidad de los cuidados, ya que aunque el esposo era su cuidador principal, ella no percibía ningún tipo de ayuda para el aseo personal, cuando apenas tenía equilibrio para permanecer sentada. Pero la cuestión no era únicamente que no recibiera ese tipo de cuidado, sino que tampoco existía ningún tipo de seguimiento por parte de los servicios sanitarios o sociales ni apoyo para formar al hombre, con el objeto de que pudiera proporcionarle los cuidados necesarios de forma adecuada.

Sin embargo, los casos españoles habría que explicarlos clarificando que las entrevistas se realizaron en el 2005, cuando todavía no se había aprobado la Ley 39/2006, del 14 de diciembre, de la Promoción de la Autonomía Personal y Atención a las personas en situación de dependencia, por lo tanto, de los entrevistados ninguna mujer recibía ningún tipo de servicio o ayuda debido a su grado de dependencia. Solamente una de ellas esperaba percibir el servicio de ayuda a domicilio tras separarse ficticiamente de su marido, quien se había empadronado en la ciudad y domicilio donde vivía un hijo de la pareja, con el fin de obtener así una mayor prestación. La falta de coordinación entre el servicio sanitario, así como la accesibilidad a los servicios y prestaciones en las situaciones de cuidados de larga duración, evidenciaba la forzada responsabilidad de la familia, y con ello, de las mujeres de la familia principalmente. Porque aunque si bien es cierto que en el caso semiindependiente el esposo era el cuidador principal, también es importante mencionar que no tenían ningún pariente o familiar que viviera cerca y además tenían dos hijos y ninguna hija.

De este modo, puede observarse cómo cuando la responsabilidad del cuidado recae en la familia, bien sea de forma forzosa (ejemplo español), como 
de forma «voluntaria» (ejemplo austriaco), en los sistemas de protección social promovían la importancia de las familias en el cuidado, de una manera u otra, reproduciendo así los roles tradicionales. Porque incluso en el caso austriaco, incluso cuando se utiliza el dinero para contratar ayuda externa al núcleo familiar, ésta suele ser femenina. En ambos casos, la falta de coordinación entre el sistema sanitario y social se hace latente repercutiendo en la calidad del cuidado ofrecida, apuntándose una necesidad en el seguimiento de los casos.

Sin embargo, los resultados apuntan la existencia de coordinación entre los Servicios Sociales y Sanitarios en Finlandia, puesto que tras el diagnóstico se elabora un plan personalizado utilizando todos los servicios y recursos disponibles para proporcionar los cuidados necesarios y de calidad a la persona enferma. Además, a través de los profesionales que atienden los casos se realiza un seguimiento de la situación, con visitas periódicas por parte de la trabajadora social del centro de rehabilitación de la provincia. Otro aspecto importante en el sistema de protección social finlandés es la prestación familiar en la que un pariente o amigo puede pasar a ser el cuidador principal y percibir un salario por ello, convirtiéndose en un cuidador formal.

Otra de las necesidades que aparece únicamente en los casos españoles, es la económica, consecuencia del sistema de protección social existente en los casos de cuidados de larga duración, en los que las familias aparecen, según los resultados preliminares, como las responsables en primera instancia de ofrecer los cuidados necesarios. El carácter de prueba de medios que tenían los servicios y prestaciones dificulta el acceso a la mayoría de los casos, además, si tenemos en cuenta que según la estructura familiar los hijos adultos permanecen en el hogar familiar hasta edades mucho más avanzadas que en otros países como Finlandia o Austria, esto empeora la accesibilidad a los mismos.

En Laponia, la falta de oferta de empleo era un factor a tener en cuenta en la opción que algunos maridos realizaban al convertirse en cuidadores formales de sus esposas a través de la prestación familiar. Por ello, y aunque la enfermedad no suponía un problema económico, la falta de trabajo en la región más despoblada del país podía vivirse como una preocupación económica y la prestación económica de cuidar a la esposa enferma una alternativa al empleo, y no una elección libre, por lo tanto, también forzada de algún modo por el contexto en el que viven. 
4.2. Las necesidades personales de los maridos o parejas de mujeres en situación de cuidados de larga duración

Las necesidades personales son entendidas en este punto como aquellas que experimentan los hombres objeto de estudio a lo largo del proceso de la enfermedad de sus esposas. Dichas necesidades no se refieren exclusivamente a los recursos necesarios para ofrecer un cuidado de calidad o para poder implicarse en el cuidado sin que de ellos dependa todo el sustento económico de la familia, sino que se refiere a las necesidades surgidas a raíz de los cambios experimentados, tanto a nivel de sentimientos y emociones como a actividades de la vida cotidiana que han tenido que modificar. Las necesidades son, en primer lugar, de vida social y tiempo libre, necesidades íntimas-sexuales, necesidad de compartir y hablar de las vivencias y sentimientos que la situación les genera, así como también necesidades de apoyo.

Las necesidades de vida social y tiempo libre han sido expresadas por todos los esposos, inclusive por aquellos que no participaban en el cuidado de sus esposas, ya que todos han experimentado cambios debido a las consecuencias de la enfermedad sufrida por sus esposas. Esta necesidad va unida al sentimiento de pérdida, como por ejemplo, por las dificultades que algunos hombres experimentan a la hora de recibir invitaciones espontáneas, así como por las dificultades por parte de algunos amigos para relacionarse con la esposa enferma. Neufeld (1998: 961-963) también mencionó en su estudio la enorme dificultad existente para los maridos cuidadores a la hora preservar su vida social, por lo que consecuentemente viven sentimiento de pérdida. Este sentimiento también va unido a los cambios producidos en la intimidad de la pareja y a la actividad sexual de la misma. Se produce una pérdida en la relación además de cambios de su amor y de la imagen que ellos tenían de sus esposas, lo que influye en sus relaciones íntimas. Las necesidades de vida íntima-sexual con sus parejas han sido estudiadas previamente por Parker y Seymour (1998: 187-191), quienes señalaron los problemas que aparecen entre los esposos cuando son cuidadores, apuntando que estos problemas se dan más entre las mujeres que entre los hombres. Sin embargo, en las entrevistas realizadas se apunta a que los problemas son más latentes entre los hombres, puesto que no se sienten cómodos teniendo relaciones sexuales con sus mujeres debido a la condición de su salud y su falta de movilidad.

También aparecen necesidades de apoyo, así como de compartir las vivencias y cambios que se están produciendo en la vida de sus familias. Sin embargo, se muestra de forma común en todos los países, pero especialmente en Finlandia y Austria, la falta de comunicación por parte de los hombres para compartir e intercambiar experiencias, y con ello recibir apoyo. Esto va unido 
a un sentimiento de resignación. McKeown, Porter-Amstrong y Baxter (2004: 223-227) señalaron que los hombres que mostraban resistencia para pedir ayuda, iban adquiriendo más y más responsabilidades del cuidado sin darse cuenta, día a día, al mismo tiempo que la enfermedad iba progresando, pero sin sentir o percibir ningún tipo de gratificación por ello, sintiéndose orgullosos del trabajo que estaban haciendo, lo que de algún modo ayudaba a que no huyeran de la situación. En este punto aparece también una preocupación acerca del destino de sus esposas en el caso de que ellos cayeran enfermos.

Junto con los sentimientos de resignación y pérdida podemos encontrar diferentes preocupaciones a lo largo del curso de la enfermedad. Un aspecto común, cuando hablamos de preocupaciones, ha sido el hecho de vivir día a día tal y como muestran otros estudios acerca del cuidado a enfermos crónicos o terminales (Lililrank, 1998). El futuro es implícitamente la preocupación principal debido al miedo de cómo adaptarse a la condición de salud de sus esposas conforme vayan empeorando, por el carácter tan impredecible de la misma. El miedo al uso de la silla de ruedas se hace patente también en la mayoría de los maridos.

La necesidad de huir se ha hecho latente en algunos de los esposos quienes han mostrado sentimientos de rechazo, sin embargo, aparece en las primeras etapas de la enfermedad por parte de algunos maridos, tal y como mencionaban McKeown, Porter-Amstrong y Baxter (2004) en su estudio, principalmente de aquellos que no participan en el cuidado de sus mujeres, como ocurre en los casos dependiente e independiente español, o en el caso semiindependiente austriaco. El sentimiento de culpa brota en los maridos que no participan en el cuidado, tal y como ocurre en el caso independiente, donde el marido no acepta la realidad ni la enfermedad de su esposa ni tampoco los cambios que ello ha significado en su vida, intentando mantener así la normalidad y evitando incluso el recibir información de la enfermedad y sus consecuencias.

El estatus marital ha sido tradicionalmente uno de los factores principales para los hombres a la hora de convertirse en cuidadores (Detinberg y Clarkberg, 2002: 873-874). Las diferencias se muestran en lo referente a los sentimientos de obligación, vinculados también al matrimonio. En Finlandia, esos sentimientos fueron expresados por los maridos, concretamente de los casos semiindependiente y dependiente, quienes expresaron que ellos se casaron delante del cura e hicieron la promesa de respetarse. De forma similar, el sentido religioso del matrimonio y la creencia del destino fue señalada por el esposo del caso semiindependiente austriaco, que remarcó que la enfermedad de su esposa es una prueba que Dios ha puesto en su camino. Sin embargo, más allá del matrimonio, la importancia de una buena relación previa entre 
las parejas, junto con la influencia de los factores de contexto, tales como los recursos y servicios accesibles de apoyo en las situaciones de cuidado de larga duración, son algunos de los factores que más influyen en la participación de los hombres en el cuidado.

\section{Conclusión}

Este estudio dispone del material necesario para abrir debate y discusión acerca de las necesidades que las personas enfermas de Esclerosis Múltiple y sus cuidadores disponen y necesitan., así como de los diferentes recursos accesibles que pueden ayudar a la mayor participación de los hombres en el cuidado, lo que viene siendo una necesidad cada vez más latente debido a los cambios que nuestra sociedad está sufriendo. Además, también abre debate sobre las necesidades personales de los hombres a la hora de desempeñar trabajos tradicionalmente relacionados con las mujeres y la influencia que las dimensiones del género pueden afectar, tanto a nivel emocional y simbólico desde el modo en que ellos viven la situación, como a nivel de poder y producción, viendo el trabajo que desarrollan influenciados por el sistema de producción social de su contexto y las relaciones de poder del mismo, fomentando la participación del hombre en el cuidado de dependientes o el hecho de que sea la mujer quien todavía se siga ocupando de esas cuestiones, relegadas al ámbito privado de la vida.

Diferentes necesidades aparecen como resultado preliminar de este estudio. En primer lugar, la falta de información en el momento del diagnóstico, lo que resulta de vital importancia para afrontar la situación y buscar los apoyos necesarios con el fin de organizar el cuidado que la mujer enferma pueda necesitar. En segundo lugar, la necesidad de que exista una coordinación entre los servicios sociales y sanitarios, primordialmente en Austria y en España, sobre todo la necesidad de un protocolo de seguimiento de los casos, que puede llegar a tener efectos para asegurar la calidad del cuidado ofrecido por los hombres, así como la formación necesaria para que la desempeñen. Y, por último, la disminución de vida social, bien sea por ser el esposo-cuidador principal, bien percibiendo una prestación económica por ello como es el caso de Finlandia, o bien porque además de participar en el cuidado de sus esposas tienen que trabajar en sus empleos habituales, lo que reduce considerablemente la vida social de los hombres, sin contar con la disminución de visitas que reciben una vez diagnosticada la enfermedad. Además, la disminución o ausencia de actividad sexual con sus parejas, siendo todavía hombres adultos y activos, es otra de las necesidades con las que tienen que lidiar los esposos. Por lo tanto, el sentimiento de pérdida es constante en sus vidas. La necesidad 
de apoyo, tanto físico como moral, también es expresada por los hombres entrevistados, quienes aprenden a vivir con resignación la vida que les ha tocado vivir, basándose para ello en creencias religiosas o en un sentimiento de obligación o culpa que les obliga a hacerlo. También, otros mencionan el amor y el cariño como la principal razón para cuidar a sus esposas. Por otro lado, muchos se van involucrando casi sin darse cuenta en las labores de cuidado que se incrementan con el tiempo, sintiéndose orgullosos de lo bien que cuidan a sus esposas pensando que nadie lo puede hacer mejor que ellos, y viviendo en una preocupación constante por el futuro cuando piensan qué sería de sus esposas si ellos cayeran enfermos. De este modo, la enfermedad de sus esposas pasa a ser el centro de sus vidas, tal y como ocurre en los casos dependiente y semiindependiente finlandés y el caso dependiente austriaco.

En consecuencia, cabe concluir diciendo que el apoyo institucional es necesario para poder ofrecer la ayuda requerida a las personas con Esclerosis Múltiple pero también a sus cuidadores, que son en muchos casos sus maridos, y que a pesar de ser minoritarios cuando hablamos de cuidados, es importante tener en cuenta que los recursos ofrecidos no deben ir encaminados a una conciliación de la vida familiar y laboral de las mujeres, sino a una corresponsabilidad de ambos. Así, son necesarios e importantes el apoyo institucional y una suficiente información y coordinación en la evolución de una enfermedad crónica para poder ofrecer cuidados de calidad. Sin embargo, no podemos olvidar las necesidades de los hombres cuidadores, que también vienen desarrollando un papel importante en situaciones de dependencia, aunque menos numeroso que las mujeres. Estas prácticas están muy influenciadas por razón de género, sin embargo se encuentran en un proceso de cambio, y es necesario prestar atención a las necesidades surgidas a este respecto. Este estudio pretende acercar las experiencias de hombres de diferentes contextos con diversos apoyos y necesidades institucionales y personales.

\section{Bibliografía}

Connell, R. W., Gender, Cambridge, Polity Press, 2002.

Detinberg, E. y M. ClarkberG, «Informal caregiving and retirement timing among men and women: gender and caregiving Relationships in late midlife», en Journal of family Issues, n. ${ }^{\circ}$ 23, 2002, pp. 857-879.

Durán Heras, M. A., Los costes invisibles de la enfermedad, Bilbao, Fundación BBVA, 2002.

EsPing-Andersen, G., The three worlds of welfare capitalism, Cambridge, Polity Press, 1990. 
FernándeZ, O. y V. E. Fernández, Esclerosis Múltiple, Málaga, Fundación Española de Esclerosis Múltiple, 2004, pp. 13-38.

FERRERA, M., Welfare State Reform in Southern Europe. Fighting poverty and social exclusion in Italy, Spain, Portugal and Greece, Londres, Reoutledge, 2005.

FInk, J., Care. Personal lives and Social Policies, Bristo, Ed. Janet Fink, 2004.

Giddens, A., Europe in the global Age, Cambridge, Polity Press, 2006, pp. 135-166.

HEARN, J., Theorizing men and men's theorizing: Varieties of discursive practices in men's theorizing of men, 1998.

KRAMER, B. J. y E. H. ThOMPSON, Men as caregivers. Theory, research and service implications, Nueva York, Springer, 2002.

KRÖGER, T. y J. SIPILÄ, Overstretched. European Families up against the demands of work and care, Oxford, Blackwell, 2005.

La PARRA CASADO, D., La atención a la salud en el hogar: Desigualdades y Tendencias, Alicante, Universidad de Alicante, 2002.

Lililrank, A., Living One Day at time. Parental Dilemmas of Managing the Experience and Care of Childhood Cancer, Stakes, National Research and Development Centre for Welfare and Health, Research Report 89, 1998.

Martínez Román, M. A., «Las familias ya no podemos más». Riesgos de exclusión social de las familias que cuidan enfermos crónicos graves», en Revista del Ministerio de Trabajo y Asuntos Sociales, n. ${ }^{\circ}$ 35, 2002 pp. 145-165.

McKeown L.P., A. P. Porter-Amstrong y G. D. BaXter, «Pressures on State Welfare in Post-industrial societies: Is more or less Better», en Making a Euroepan Welfare State? Convergences and Conflicts over European Social Policy?, Taylor \& Gooby (eds.), 2004, pp. 17-35.

NeUfELD, A., «Men as caregivers: reciprocal relationships or obligation?», Journal of Advanced Nursing, 28(5), 1998, pp. 959-968.

PARKER, G. y J. SEYMOUR, "Male carers in marriage», en Re-examining feminist analysis of informal care, J. Popay, J. Hearn y J. Edwards, Routledge, Men Gender Divisions and Welfare, 1998.

PeAse, B. y K. Pringle, «Introduction: Studying Men's Practices and Gener Relations in a Global Context» En A man's world? Changing men's practices in a globalized world, B. Pease y K. Pringle (eds.), Reino Unido y Londres, Zed Bools Ltd., 2001, pp. 1-17.

Sanders, S., C. Morano, y S. C. Corley, «The expressions of Loss and Grief among Male Caregivers of individuals with Alzheimer's Disease», en Journal of Gerontological Social Work, 39 (4), 2002.

StOller, E. P., «Theoretical Perspectives on Caregiving men» en Men as Caregivers: Theory, research and service implications, Thompson y Kramer, Nueva York, Springer, 2002, pp. 51-68. 
StONE, R. I., «Long-Term Care Workforce Shortages: Impact on Families», en Family Caregiver Alliance, Policy Brief, 3, [en línea], 2001, <http://www.bjbc. org/content/docs/LTC_Workforce_Shortages.odf>. [Consulta: 20/11/2007].

ZECHNER, M., "Care of Older Person in Transnational Settings», en Journal of Aging Studies, 22, 2008, pp. 32-44. 